\section{Cochlear Implantation and Re- petitive 3.0 Tesla MRI Scans in a Multiple Sclerosis Patient with Congenital Deafness-A Case Report}

\section{Buchberger $\mathrm{AMS}^{1^{*}}$, Pickhard $\mathrm{A}^{1}$, Storck $\mathrm{K}^{1}$, Strenger $\mathrm{T}^{2}$ and Stark $T^{3}$}

${ }^{1}$ Department of Otorhinolaryngology, Head \& Neck surgery, Technical University Munich, Munich, Germany

${ }^{2}$ Department of Otorhinolaryngology, Head \& Neck surgery, Klinikum Augsburg, Augsburg, Germany

${ }^{3}$ Department of Otorhinolaryngology, Head \& Neck surgery, HELIOS Hospital west of Munich, Munich, Germany

\section{Abstract \\ Objective}

In the past, Magnetic Resonance Imaging (MRI) in patients with Cochlear Implants $(\mathrm{Cl})$ used to be problematic. This was due on the one hand to magnet or electrode displacement or disturbances as a result of the magnetic field induced by MRIs, and on the other hand due to scan interruption following patients' pain and discomfort.

Design

In this case report we present the case of a 20-year old female patient with both-sided deafness since childhood, who expressed the wish for Cls. The patient had been recently diagnosed with multiple sclerosis meaning she would be needing recurrent MRI scans.

\section{Results}

The patient was implanted with a novel MRI conditional system produced by MED-El and received postoperative MRI head and vertebral scans with no side effects.

\section{Conclusion}

Cls do not exclude repetitive MRI scans up to 3 Tesla anymore. The risk of side effects as a result of the magnetism has been diminished.

*Corresponding author: Buchberger AMS, Department of Otorhinolaryngology Head \& Neck surgery, Technical University Munich, Munich, Germany, Tel: +49 08941402694; E-mail: maria.buchberger@tum.de

Citation: Buchberger AMS, Pickhard A, Storck K, Strenger T, Stark T (2017) Cochlear Implantation and Repetitive 3.0 Tesla MRI Scans in a Multiple Sclerosis Patient with Congenital Deafness-A Case Report. J Otolaryng Head Neck Surg 3: 012.

Received: June 02, 2017; Accepted: August 07, 2017; Published: August 21, 2017

\section{Introduction}

High-resolution Computed Tomography (CT) and high-resolution Magnetic Resonance Imaging (MRI) are standard examinations that precede the insertion of a cochlear implant, a proven treatment for severe to profound bilateral hearing loss [1]. Imaging has an important role in deciding whether a cochlear implant is a therapeutical option, providing realistic preoperative counseling, and predicting the postoperative outcome [2].

So far, MR imaging post implantation have been problematic and is only performed if considered absolutely necessary and with very strict indications and specific preparatory measures. Due to the magnetic field induced during an MRI, electrode and magnet dislocation as well as burning sensations and severe pain has been extensively reported [3].

But what if the patient needs repetitive MR imaging to monitor underlying diseases like multiple sclerosis, tumor diseases or progressing degenerative conditions?

A novel CI system, called Synchrony, has been introduced by MED-EL GmbH (Innsbruck, Austria) and has been approved for regular and repetitive MR imaging up to 3.0 Tesla without the need to remove the inner magnet to avoid typical side effects. The main feature in this new system is the rotating implant magnet that can adjust its position to the magnetic field induced during MRIs up to three Tesla (T). The safety guidelines for the Synchrony CI system and MRIs are similar to those for other CI models that have been in use for years. Before patients enter the MRI room, all external components of the implant system have to be removed. An additional supportive head bandage can be placed over the implant for field strengths of 1.0, 1.5, and $3.0 \mathrm{~T}$. The head should be oriented straight and an inclination to one side should be avoided. For up to $3.0 \mathrm{~T}$, the normal operating mode can be used. For 3.0 T scans the SAR limit should not exceed $1.6 \mathrm{~W} / \mathrm{kg}$ for head imaging or $1.0 \mathrm{~W} / \mathrm{kg}$ for whole-body scans below $35 \mathrm{~cm}$ from the vertex, and $2.0 \mathrm{~W} / \mathrm{kg}$ for whole-body scans above 35 $\mathrm{cm}$ from the vertex to avoid possible heating at the electrode contacts. If these precautions are followed the incidence of MRI associated side effects seems to be extremely low and to our knowledge no side effects are reported in literature for the CI Synchrony system.

The following case study will report the case of a young female patient with newly diagnosed multiple sclerosis and the need for bilateral CIs due to congenital deafness. The new CI system was successfully implanted in the patient, allowing for recurrent MRIs needed to monitor the status of the underlying neurologic disease.

\section{Case Report}

A 20-year old female patient from Qatar born in 1995, presented to our Ear-Nose and Throat (ENT) outpatient clinic with vertigo and dizziness which had persisted for one year. She was diagnosed with severe bilateral sensory neural hearing loss at birth and underwent a cochlear implantation in 1999 on the right side. Her speaking ability was properly developed and verbal communication therefore possible. The right sided implant had been positioned rather cranial and the patient asked for a revision with a correction of the location as well as an implantation on the left side. 
The patient explained that her vertigo had been worsened progressively for the past year and that she had experienced intermittent numbness and paresthesia in all four extremities.

\section{Clinical Findings}

\section{ENT check}

On inspection and palpation the CI system on the right temporal region was in situ.

The ear microscopy showed free ear ducts and intact ear drums with no signs of middle ear infusion or infection. No spontaneous nystagmus, provocative nystagmus or vibration nystagmus could be observed through the frenzel lenses. The rest of the clinical ENT examination met expectations according to the patient's age and didn't include any notable findings.

Several clinical and objective findings were notable concerning the vertigo. The Vestibule Oculography (VOG) revealed a bilateral non compensated loss of vestibular function. In the electronystagmography no spontaneous nystagmus was noted. Both the rotation test and the caloric provocation with $40^{\circ} \mathrm{C}$ temperate water showed a lack of response on both sides. The optokinetic testing was symmetric, with smooth visual sequences with no signs of a saccadic pursuit eye movement.

The patient was not able to perform the Romberg's test and Unterberger's test since she could not stand up without help. The dix-hallpike test was negative, ruling out a paroxysmal benign positional vertigo.

\section{Pre-operative CT scan}

A CT scan was performed as pre-operative planning imaging. Due to the CI in situ on the right site, which was not MRI compatible, it was not possible to perform an MRI. The CT showed free cerebellopontine angles on both sides, a free and intact internal ear duct and no signs of tumorous lesions. The presence of the CI beam hardening artifacts limited the assessment of the CT scan though.

\section{Audiometry}

The Brainstem Evoked Response Audiometry (BERA) offered no reproducible stimulus response on either side.

The pure tone audiogram showed a remaining low frequency hearing capacity on the right side from 125 to $250 \mathrm{Hertz}(\mathrm{Hz})$ at $90-105$ decibel $(\mathrm{dB})$, and a pantonal hearing curve between 20-30 dB with the cochlea implant. On the left side, the remaining low frequency hearing capacity between $125-250 \mathrm{~Hz}$ was at $60 \mathrm{~dB}-90 \mathrm{~dB}$.

The transient evoked otoacoustic emission showed no function of the external hair cells on either side.

The tympanogram was interpreted as a type a bilateral, with regular peaks. On the right side the stapedius reflexes could be triggered with the CI switched on whereas on the left side no reflexes were seen.

\section{Neurological findings}

Prior to any further evaluation or surgery the patient was examined by a neurologist. Following his clinical finding and a lumbal puncture he diagnosed a Relapsing Remitting Multiple Sclerosis (RRMS) with ataxia, limited vision and impaired motoric movement with an inability to walk but no signs of spasticity. The lumbar puncture was positive for oligoclonal bands and showed intrathecal immunoglobulin $G$ production. Rheumatoid diseases of any kind were ruled out.

\section{Therapy}

The neurologist started the patient on a steroid pulse therapy and gilenya and the patient's condition improved considerably. As for the $\mathrm{CI}$, it was suggested the patient should have the $\mathrm{CI}$ on the right side removed to allow for regular MR Imaging, which is highly recommended to monitor the neurological disease.

Since the patient had subjective difficulties with the right-side cochlear implant and wished for an implantation on the left side, we decided in consultation with the treating neurologist to explant the right side, perform an intraoperative MRI to document the state of the white matter lesions in the brain and reimplant the right side with a new, previously selected system. The left side would then simultaneously be implanted with the same new system as the right side.

The surgery was performed in intubation narcosis according to the interdisciplinary consented plan.

First the old scar was circumcised and the old implant was freed from surrounding tissue on the right side. The active and passive electrodes were secured and the implant recovered. After the wound was closed and covered, an MRI was performed.

The intraoperative scan in figure 1 shows multiple T2 supra-and infratentorial hyper intensive cerebral parenchymal lesions in the medulla oblongata, and in the pons as well as bilaterally in the cerebrella peduncula, cerebrellar hemisperes and mesencephal, in the bar, and subcortical in the medullary layer. After contrast enhancement, a barrier defect at the bar as well as frontal on the left side was observed. The cerebellum-pons angle showed no pathological changes, especially no signs of a tumor such as a vestibular schwannoma. The inner ear anatomy seemed regular and correct on both sides and the facial and vestibulocochlear nerves could also be tracked well.

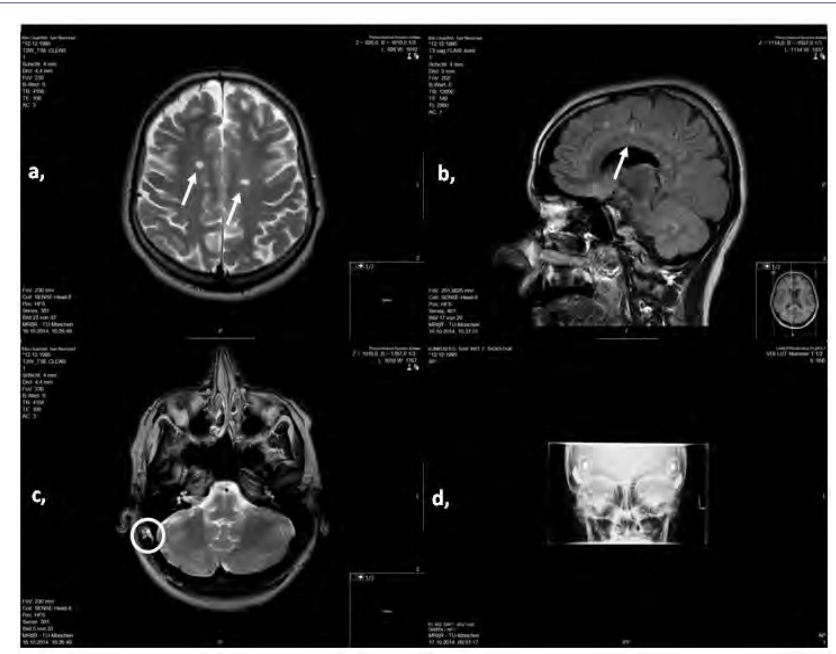

Figure 1: Intraoperative MRI and postoperative X-Ray.

T1 and T2 weightings show MS-typical white matter lesions (white $\uparrow$ ) in a, and $b, . c$, shows dditionally fluid retention in the right mastoid $(0$ : operative field). $d$, is the postoperative transorbital X-Ray for location control.

With this MRI, the typical findings for the diagnosis of MS were revealed and any hearing nerve impairment or tumors were ruled out. Subsequently, the reimplantation on the right side as well as implantation on the left side was both carried out with continuous neuromonitoring of the facial nerve on both sides. 
The implantation was performed as previously described by our clinic $[4,5]$. We chose the synchrony standard processor Opus 2 with a $\mathrm{D}$ coil with a magnet force 4 . After successfully completing surgery and a postoperative transorbital X-ray (Figure 1) showing the electrodes in situ the patient was dismissed from the floor on the fourth post surgical day in good condition and with good wound healing.

The first CI adaption was performed by our engineers 4 weeks later in 5 separate visits. A postsurgical tone audiogram reconfirmed the remaining and unchanged deep frequency hearing capacity and a pantonal hearing curve with the CI on the right side and with 20-30 $\mathrm{dB}$ and $30-40 \mathrm{~dB}$ on the left side (Figure 2).

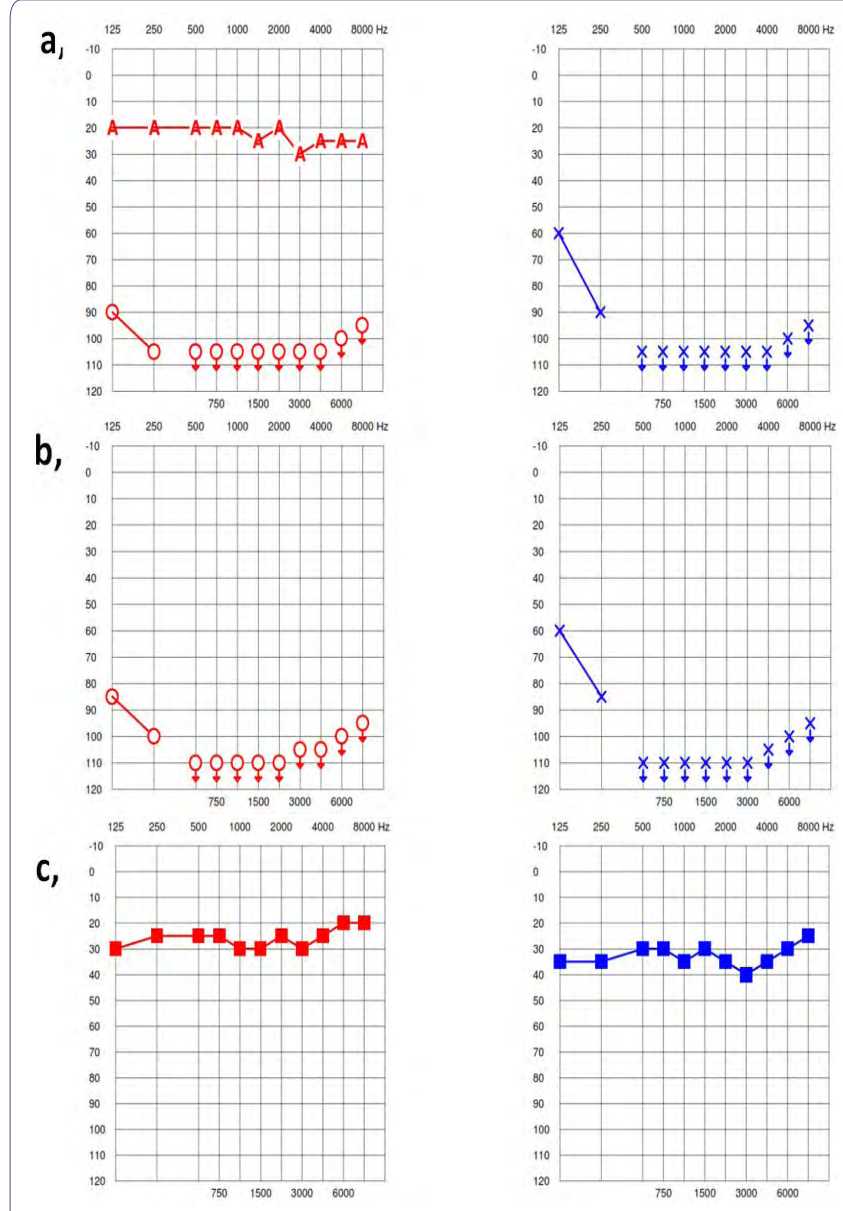

Figure 2: Pre-and Postsurgical Pure Tone Audiometry.

a) shows the pre-surgical pure tone audiometry with and without the $\mathrm{Cl}$ on the right side and a remaining hearing capacity for the deep frequencies. b) shows the postsurgical audiometry with the preserved remaining hearing and c) the improved hearing on both sides with the Cls.

After the surgery MRI scans had to be done to reevaluate the MS status. These were tolerated with no signs of electrode or magnet displacement, discomfort or pain (Figure 3). The manufacturer's instructions, explained in the introduction, were followed. Although the extinction artifacts are obvious as regards the hemisphere area, the treating neurologist was satisfied with the image quality, as the white matter lesions were predominantly spinal at that time. In case of the need for better quality imaging of the hemispheres, the magnet could be removed in local anesthesia beforehand.

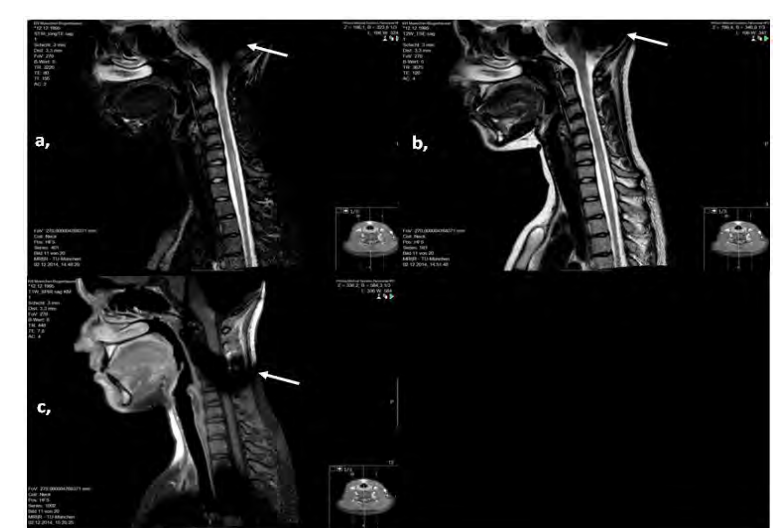

Figure 3: Post-surgical MRI of the spine with the synchrony $\mathrm{Cls}$ on both sides.

Post-surgical spine MRI performed elsewhere. All images in sagittal plain, showing the extinction artifacts (white $\uparrow$ ) due to the implanted Cls. Picture a) is a STIR image, picture b) is in T2W Sequence and picture c) is a T1W SPIR Sequence. One can see signal alteration as a pathologic finding all along the cervical spine for $\sim 7 \mathrm{~cm}$.

Since the surgery the patient has only been in the country for checkups and has tolerated yearly performed MRIs well. The hearing capacity has not changed and the patient is very satisfied with the hearing and communication situation.

\section{Discussion}

The need for repeated MRIs used to be a contraindication for the implantation of cochlea implants. Even when MRI instructions by the different manufactures are followed, the risk of magnet dislocation, electrode dislocation or severe injuries and pain was still a serious problem [6]. Kim et al., [7] retrospectively analyzed 18 patients with CIs undergoing MRIs with a $3.0 \mathrm{~T}$ scanner, 12 of whom had head scans. Five patients were unable to complete the scan due to pain, one experienced a magnet displacement and another had to be surgically revised due to magnet problems. Hassepass et al., reviewed 1706 CI cases from 2000 to 2011 and reported that about 1.23\% (21 patients) had revision surgeries with internal magnet removal or replacement. Of these surgeries, $47.62 \%$ were caused by $1.5 \mathrm{~T}$ MRIs all between 2009 and 2011 [6]. All events had occurred despite medical staff having followed the current safety recommendations concerning MR imaging in patients with cochlear implants. The total number of MRIs for this cohort was not published and before 2009 all patients had to undergo magnet explanation prior to the MR imaging. In a second report with 2027 patients the percentage of magnet dislocations due to $1.5 \mathrm{~T}$ MRIs was reported as $0.59 \%$ (12 patients) of all patients, causing $52.2 \%$ of revision surgeries. Eight patients had their internal magnets removed before an MRI was performed [6]. In a series of 300 patient with CIs, 30 patients needed an MRI due to other diseases such as parotid gland tumors or cervical discus prolaps. None of these patients had the internal magnet removed and no adverse events were reported by Baumgartner et al. [8]. Although these results show that adverse events are rare, they are still to be considered as a serious threat to patients' health and have to be avoided. All of the above studies were carried out with $1.5 \mathrm{~T}$ scanners. These might not deliver images with the resolution needed to monitor neurologic diseases [9]. 3.0 T scanners have had a growing market share for years and nowadays they are often favored due to the better quality of the images and faster results [10]. Most hospitals and outpatient radiologists now offer 3.0 T scans on a daily basis. For the diagnosis of MS, the MRI is considered to be the diagnostic gold standard and in some countries of even of higher 
value than spinal fluid examinations (Current S2e German Guidelines for Diagnosis and Therapy of Multiple Sclerosis, register number 030050).

Multiple sclerosis per se is no contraindication for the implantation of a CI [11] but has been a clear limitation due to the complication regarding MRIs. To our knowledge we are the first clinic to successfully implant a patient with multiple sclerosis with the new CI model Synchrony of MED-EL, making repetitive MRI scans possible and side effects less likely thanks to a rotating internal magnet.

\section{References}

1. Stark T, Helbig S (2011) [Cochlear implantation: a changing indication]. HNO 59: 605-614.

2. Vogl TJ, Tawfik A, Emam A, Naguib NN, Nour-Eldin A, et al. (2015) Pre-, Intra- and Post-Operative Imaging of Cochlear Implants. Rofo 187: 980-989.

3. Hassepass F, Stabenau V, Arndt S, Beck R, Bulla S, et al. (2014) Magnet dislocation: an increasing and serious complication following MRI in patients with cochlear implants. Rofo 186: 680-685.

4. Stark T, Niedermeyer HP, Knopf A, Sudhoff H (2011) Surgical technique for implantation of the MED-EL SONATATI. ORL J Otorhinolaryngol Relat Spec 73: 196-200.
5. Niedermeyer HP, Jaschke K, Holzapfel S, Stark T (2011) B033 First results in cochlear implantation using the new MED-EL CONCERTO device. International Journal of Pediatric Otorhinolaryngology 75: 16

6. Hassepass F, Stabenau V, Maier W, Arndt S, Laszig R, et al. (2014) Revision surgery due to magnet dislocation in cochlear implant patients: an emerging complication. Otol Neurotol 35: 29-34.

7. Kim BG, Kim JW, Park JJ, Kim SH, Kim HN, et al. (2015) Adverse events and discomfort during magnetic resonance imaging in cochlear implant recipients. JAMA Otolaryngol Head Neck Surg 141: 45-52.

8. Baumgartner WD, Youssefzadeh S, Hamzavi J, Czerny C, Gstoettner W (2001) Clinical Application of Magnetic Resonance Imaging in 30 Cochlear Implant Patients. Otology \& Neurotology 22: 818-822.

9. Wood R, Bassett K, Foerster V, Spry C, Tong L (2011) 1.5 Tesla Magnetic Resonance Imaging Scanners Compared with 3.0 Tesla Magnetic Resonance Imaging Scanners: Systematic Review of Clinical Effectiveness. CADTH Technol Overv 2: 2201.

10. Willinek WA, Kuhl CK (2006) 3.0 T 3.0 T neuroimaging: technical considerations and clinical applications. Neuroimaging Clin N Am 16: 217-228.

11. Shanbhag J, Vaid N (2010) Cochlear implantation in a profoundly deaf patient who also suffers from multiple sclerosis--a case study. Cochlear Implants Int 11: 117-123. 